\title{
DETERMINACIÓN DE 222Rn POR ADSORCIÓN EN CARBÓN ACTIVADO Y SU IMPACTO RADIO AMBIENTAL EN DOS INSTITUCIONES EDUCATIVAS DEL DEPARTAMENTO DE PUNO (MACUSANI)
}

\author{
H. Chui ${ }^{\mathrm{a}}$, J. Osores ${ }^{\mathrm{b}}$ y P. Olivera ${ }^{\mathrm{b}}$ \\ ${ }^{a}$ Universidad Nacional Mayor de San Marcos (UNMSM). Apartado postal 14-0149, Lima 14 - Perú. \\ ${ }^{b}$ Seguridad Radiológica-Instituto Peruano de Energía Nuclear (IPEN)
}

\begin{abstract}
Resumen
Un enemigo de la salud que es temible, invisible y silencioso se prolifera en ambientes cerrados. Un gas inerte denominado radón, es producto del uranio ${ }^{238} \mathrm{U}$, uranio ${ }^{235} \mathrm{U}$ y torio ${ }^{232} \mathrm{Th}$ y es considerado cancerígeno por la Organización Mundial de la Salud (OMS). Por los súbitos cambios climáticos en la sierra de Puno, los estudiantes se ven obligados realizar sus actividades en ambientes cerrados. El distrito Macusani en Puno es el distrito uranífero mas importante del Perú. Puesto que el uranio se desintegra en isótopos radiactivos tales como ${ }^{218} \mathrm{Po},{ }^{214} \mathrm{~Pb},{ }^{214} \mathrm{Bi},{ }^{214} \mathrm{Po} \ldots{ }^{206} \mathrm{~Pb}$, que son muy peligrosos. El presente trabajo tiene como objetivo el cálculo de la concentración de radón en ambientes educativos cerrados y el impacto que puede generar en los estudiantes de las instituciones educativas. Todo este estudio fue realizado en Macusani, analizado con técnicas de espectroscopia gamma y canister de carbón activado y con el apoyo de la Fluorescencia de rayos X de los laboratorios del Instituto Peruano de Energía Nuclear (IPEN) con el asesoramiento técnico y de capacitación del área de Seguridad Radiológica.
\end{abstract}

Palabras claves: Radón, Puno, Uranio, Radioactivo.

\begin{abstract}
An enemy of the health is invisible, frightful and quiet exists that appropriately is proliferated in closed environments. An inert gas denominated radon, which is product of uranium ${ }^{238} \mathrm{U}$, uranium ${ }^{235} \mathrm{U}$ and thorium ${ }^{232} \mathrm{Th}$ and is considered cancerigenic by the World Organization of the Health (WHO). By the abrupt climatic changes in the Puno zone (Macusani), the pupils of the schools are forced to study in closed rooms. Macusani is the most important district of Peru which produces uranium. Uranium disintegrates in its daughters ${ }^{218} \mathrm{Po},{ }^{214} \mathrm{~Pb}$, ${ }^{214} \mathrm{Bi},{ }^{214} \mathrm{Po} \ldots{ }^{206} \mathrm{~Pb}$, which are radioactive and dangerous. For that reason the present work of investigation focuses on the calculation the concentration of radon, in educative environments and the impact that it generates on the students. This study was made in Macusani. The analysis (with techniques of gamma spectroscopy and canister of activated charcoal) was done with the support of the $\mathrm{x}$-rays fluorescence in the laboratories of the Peruvian Institute of Nuclear Energy (IPEN) with technical support enabled by the IPEN area of Radiological Security.
\end{abstract}

Keywords: Radon, Puno, Uranium, Radioactive.

\section{Introducción}

El ${ }^{222} \mathrm{Rn}$ es la fuente más importante de la radiación natural. Se ha estimado que el ${ }^{222} \mathrm{Rn}$ y sus descendientes de corto periodo como el ${ }^{218} \mathrm{Po}$,
${ }^{214} \mathrm{~Pb},{ }^{214} \mathrm{Bi},{ }^{214} \mathrm{Po} . . .{ }^{206} \mathrm{~Pb}$ (este ultimo es estable), contribuyen con las tres cuartas partes de la dosis efectiva anual recibida por el hombre de las fuentes terrestres naturales, y es responsable de aproximadamente la mitad de la dosis recibida

E-mail:P130066@unmsm.edu.pe 
de la totalidad de las fuentes [1]. Debido a esto es importante realizar el control de niveles de radón en lugares donde labora el hombre.

El Uranio es ampliamente distribuido en la naturaleza y es un contaminante menor de todas las rocas, arenas y el suelo. La cantidad típica del uranio en la tierra o suelo esta comprendida entre 1 y 4 Partes por millón, por ende el suelo y las rocas contienen una parte proporcional de ${ }^{226} \mathrm{Ra}$. Este radio se transforma a una razón constante, y dentro de sus descendientes radioactivos se encuentra el ${ }^{222} \mathrm{Rn}$. Que mantiene una actividad constante. El ${ }^{222} \mathrm{Rn}$ es el único elemento en la serie de decaimiento del Uranio, que es gas a temperaturas ordinarias (el ${ }^{222} \mathrm{Rn}$ se licua a $-62{ }^{\circ} \mathrm{C}$ y que es el miembro mas pesado dentro de la familia química de los gases monoatómicos inertes). Ya que todas las rocas y suelo son ligeramente porosos el ${ }^{222} \mathrm{Rn}$, logra difundirse a través de ellos a ala superficie de la corteza terrestre [2]. Esta es la razón por la cual puede estar presente en el aire o en el agua a la cual los seres vivos siempre tenemos acceso, es un emisor de partículas alfa de 5,499 MeV, el tiempo de vida media del ${ }^{222} \mathrm{Rn}$ es de 3.82 días lo cual es suficientemente grande.

En los últimos años la atención hacia las emanaciones de ${ }^{222} \mathrm{Rn}$ ambiental ha crecido drásticamente. Hasta antes de la década de los años sesenta se pensaba que las elevadas concentraciones de radón en interiores era un asunto asociado a la inadvertida presencia de deshechos industriales y residuos de minas en construcciones aledañas a estos. Observaciones efectuadas en Canadá y Estados Unidos en los años ochenta encontraron una alta incidencia de habitaciones ordinarias con niveles elevados de radón. Esto llamó la atención a la comunidad científica de este fenómeno, ahora ampliamente conocido. Una cuestión mas significativa es que el nivel estimado de riesgo de salud asociado con los niveles promedio del radón en interiores es mucho mas alto que los niveles de los otros contaminantes ambientales cancerígenos [3].

La Relación entre la exposición a niveles elevados de radón en las minas de uranio y el padecimiento de cáncer pulmonar esta firmemente establecida. El efecto del radón a los niveles encontrados en los lugares comunes de asentamiento humano es incierto aun existen ciertas diferencias entre la exposición al radón en las minas y en los ambientes normales de las habitaciones, escuelas, hospitales, fabricas, etc y también entre los mineros y la gente común, las concentraciones de radón en interiores dependen de la geología, estructura, construcción. Para la estimación de los riesgos, los métodos que actualmente se usan son: Epidemiología, dosimetría modelada, estudios del control de caso y estudio de la transformaciones de la célula, el interés principal esta centrado en los problemas hematológicos debidos al Radón[4]. Nuestro país posee una gran riqueza uranífera, ubicado en el departamento de Puno provincia de Carabaya distrito de Macusani, que en un informe reciente preparado bajo el auspicio del OCDE (Organismo de Cooperación para el Desarrollo Económico) para la energía nuclear y el OIEA, se señala que existe posibilidades de encontrar 6000 toneladas de uranio. El distrito de Macusani de la provincia de Carabaya se encuentra ubicado a $4450 \mathrm{msnm}$. En la zona norte del departamento de Puno, en la vertiente oriental de la Cordillera de los Andes, entre las coordenadas: $14^{\circ} 03^{\prime} 51^{\prime \prime}$ de latitud sur; y $70^{\circ} 25^{\prime}$ $45^{\prime \prime}$ de longitud oeste del meridiano de Greenwich; la capital de la provincia se encuentra ubicada a $256 \mathrm{Km}$. de distancia de la capital del departamento de Puno. Pues presumimos que el potencial de ${ }^{222} \mathrm{Rn}$ en Macusani es alto debido a la concentración alta de uranio, y para ello se hizo diversos tipos de muestreo in situ y en laboratorio (IPEN). El propósito de este trabajo es la determinación de la concentración del ${ }^{222} \mathrm{Rn}$ en el aire, mediante la adsorción en carbón activado y la posterior medición de las hijas, en espectrometría Gamma.

\section{Metodología}

En espectrometría gamma la actividad de un radionúclido viene dada por la expresión: [5]

$$
A=\frac{N}{\varepsilon_{p} p_{\gamma} T} ;
$$

Donde $N$ es el número neto de cuentas en el fotopico de interés, $T$ es el tiempo de recuento y $P_{\gamma}$ es la probabilidad de emisión. Para obtener la eficiencia de fotopico en una muestra real $\left(\varepsilon_{p}\right)$, definiremos el factor de corrección como: $f=\varepsilon_{p} / \varepsilon_{c}$ donde $\varepsilon_{\mathrm{c}}$ es la eficiencia de fotopico en la muestra de calibración empleada, fosfato 
roca, una matriz de calibración sólida. Asumiendo que los fotones emitidos por la muestra inciden normalmente en el detector (geometría cilíndrica), el número de fotones transmitidos sin interaccionar en la muestra vendrá dado por la ecuación de autoabsorción:

$$
n_{s}=n_{0}\left[\frac{1-e^{-\mu \rho h}}{\mu \rho h}\right\rfloor ;
$$

donde $\mu$ es el coeficiente de atenuación másico $\left(\mathrm{mac}, \mathrm{cm}^{2} \mathrm{~g}^{-1}\right), \rho$ es la densidad aparente $\left(\mathrm{gcm}^{-3}\right), h$ es la altura de muestra seleccionada y $n_{0}$ es el número de fotones que originados en la muestra, viajan directamente hacia el detector (número de fotones que teóricamente alcanzarían el detector en ausencia de autoabsorción). De este modo las eficiencias de fotopico $\varepsilon_{\mathrm{p}}$ y $\varepsilon_{c}$. (muestras real y de calibración, respectivamente) vendría dada por [5]:

$$
\left.\varepsilon_{p}=\varepsilon_{0} \mid \frac{\left.1-e^{-\mu_{p} \rho_{p} h}\right\rceil}{\mu_{p} \rho_{p} h}\right\rfloor ; \varepsilon_{c}=\varepsilon_{0}\left\lfloor\frac{1-e^{-\mu_{c} \rho_{c} h}}{\mu_{c} \rho_{c} h}\right\rfloor ;
$$

donde $\varepsilon_{0}$ es la eficiencia de fotopico en ausencia de autoabsorción y sólo depende de la energía y de la geometría de medida, es decir, $\varepsilon \rightarrow \varepsilon_{0}$ si $\mu \rightarrow 0$, en ambos casos. Entonces, teniendo en cuenta (3), el factor de corrección $f=\varepsilon_{\mathrm{p}} / \varepsilon_{\mathrm{c}}$ puede escribirse como: [5]

$$
f=\left\lfloor\frac{1-e^{-\mu_{p} \rho_{p} h}}{\mu_{p} \rho_{p} h}\right\rfloor\left[\frac{\mu_{c} \rho_{c} h}{1-e^{-\mu_{c} \rho_{c} h}}\right\rceil ;
$$

donde las densidades aparentes de las muestras reales y de calibración son conocidas, $h$ es la altura de muestra y $\mu$ puede obtenerse a través de la fórmula de Bragg: [5]

$$
\mu=\sum \omega_{i} \mu_{i} ;
$$

donde $\omega_{i}$ es la fracción en peso de cada componente en la muestra de calibración. El coeficiente de atenuación másico ( $\mathrm{mac}$ ) para las muestras reales, $\mu_{\mathrm{p}}$, será conocido ya que la composición elemental del suelo será determinada.

Puede usarse un valor medio para el coeficiente de atenuación másico representativo del área de estudio. El colector empleado en este trabajo, aparece en la Fig 2.1 y en él se colocan, aproximadamente, 20 gramos de carbón activado.

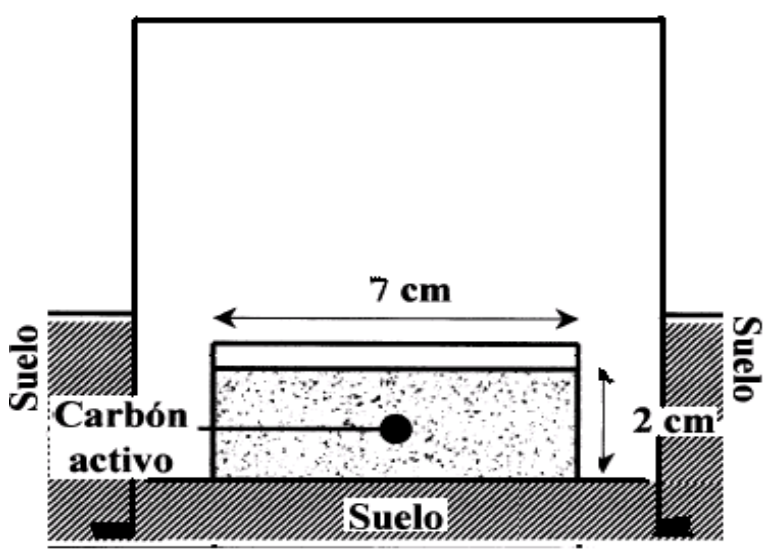

Figura 2.1. Este es un esquema representativo de la forma en que trabajó con cartuchos y portacartuchos de carbón activado.

A continuación, el carbón activado se sella con unas tapas que impiden la salida del Rn, el cual alcanzará el equilibrio con sus descendientes a las 4 horas y estará preparado para su análisis en el laboratorio. La cantidad de ${ }^{222} \mathrm{Rn}$ absorbida en el carbón activado es determinada por espectrometría gamma a partir del fotopico de $609-\mathrm{keV}$ del ${ }^{214} \mathrm{Bi}$, descendiente del ${ }^{222} \mathrm{Rn}$. El sistema de espectrometría gamma está constituido por un detector semiconductor de $\mathrm{Ge}$ intrínseco, coaxial, tipo reverse (ReGe). Una vez retirada cada cartucho de carbón y que el ${ }^{222} \mathrm{Rn}$ teóricamente alcanzó el equilibrio radiactivo con sus descendientes de vida corta, la determinación de la actividad del ${ }^{222} \mathrm{Rn}$ se llevó a cabo por espectrometría gamma a partir del recuento de las emisiones registradas en la zona del espectro que abarca el fotopico de $609 \mathrm{KeV}$ del ${ }^{214} \mathrm{Bi}$, y ${ }^{214} \mathrm{~Pb}$. La determinación de la exhalación $(\mathrm{E})$ en $\mathrm{Bq} \mathrm{m}^{-2} \min ^{-1}$ se llevará a cabo mediante la siguiente expresión general [6]

$$
E=\frac{N \lambda^{2} e^{\lambda t d}}{\varepsilon S\left(1-e^{-\lambda t c}\right) C F(h)},
$$

donde:

$\mathrm{N}$ : número neto de cuentas registrado en la zona del espectro analizada,

$\varepsilon$ : eficiencia de detección del método espectrométrico en $\mathrm{cpm} / \mathrm{Bq}$.

$\lambda$ : constante de desintegración radiactiva del ${ }^{222} \mathrm{Rn}$ expresada en minutos- 1 .

tc: tiempo de contaje empleado.

td: tiempo transcurrido desde el final de la exposición del cartucho de carbón hasta el comienzo de su contaje, (tc y td expresados ambos en minutos). 
$\mathrm{CF}(\mathrm{h})$ : es un factor de calibración que para un tiempo de exposición dado depende fundamentalmente de la humedad retenida en el carbón [6].

S: área del cartucho, área de la base del cartucho en contacto con el suelo.

\section{Resultados y Discusión}

A continuación se muestra los resultados obtenidos del estudio realizado, se trabajó paralelamente en dos Instituciones educativas:

Institución educativa Secundaria Mariano Melgar Ayaviri, se encuentra en la provincia de Melgar del departamento de Puno zona que esta ligeramente alejada del yacimiento uranífero. Institución educativa Secundaria José Macedo Mendoza Macusani, se encuentra en la provincia de Carabaya del departamento de Puno, Zona que se encuentra muy próximo al yacimiento de Uranio.

\begin{tabular}{|l|c|c|}
\hline \multicolumn{3}{|c|}{ RESUMEN } \\
\hline Grupos & AYAVIRI & MACUSANI \\
\hline $\begin{array}{l}\text { Concentración; } \\
\text { Bq/m }\end{array}$ & 21.074 & 105.323 \\
\hline $\begin{array}{l}\text { Promedio, horas en } \\
\text { casa diarias. }\end{array}$ & 14.07 & 15.68 \\
\hline $\begin{array}{l}\text { Promedio; horas en } \\
\text { clase diarias. }\end{array}$ & 5.53 & 6.11 \\
\hline $\begin{array}{l}\text { Dosis Efectiva por } \\
\text { permanencia en clase }\end{array}$ & $0.047 \mathrm{mSv}$ & $0.264 \quad \mathrm{mSv}$ \\
\hline $\begin{array}{l}\text { Concentración de } \\
\text { Uranio en el suelo; } \\
\text { ppm (FRX) }\end{array}$ & 4 & 23.3 \\
\hline
\end{tabular}

\section{Conclusiones}

Puno se encuentra dentro de un yacimiento uranífero, y teniendo en cuenta este factor se llevo a cabo el estudio, con el propósito de identificar la concentración y su impacto ambiental en las instituciones educativas mencionadas, de acuerdo a los análisis por espectrometría Gamma de las muestras obtenidas se encontró que para la IESMMA (Ayaviri) la concentración de ${ }^{222} \mathrm{Rn}$ es de $21.074 \mathrm{~Bq} / \mathrm{m}^{3}$ y para la IESJMM (Macusani) es de 105.323 $\mathrm{Bq} / \mathrm{m}^{3}$ estos resultados se encuentran por debajo del limite permisible establecido por la OMS Y EPA. En cuanto a la dosis efectiva percibida por los alumnos de acuerdo con la tabla de conversiones obtenemos que para la IESMMA(Ayaviri) la dosis efectiva es de 0.047 $\mathrm{mSv}$ y para la IESJMM(Macusani) es de 0.264
mSv estos resultados son un estimado, solo para el tiempo que están en clases durante un año, los resultados se encuentran por debajo del límite permisible establecido por la OMS Y EPA. El Hecho de que se haya encontrado resultados de bajo de los límites permisibles no quiere decir que no hay riesgo de cáncer, este dato solo nos dice que el riesgo puede ser controlado.

La concentración de uranio y torio en un suelo normal se encuentra entre 4 a $7 \mathrm{ppm}$ [3] y en una muestra extraída del muro de la institución educativa José Macedo Mendoza, Analizada por la Técnica de Fluorescencia de rayos X, dan resultados de $23.3 \mathrm{ppm}$ de uranio y de $19.5 \mathrm{ppm}$ de torio.

\section{AGRADECIMIENTOS}

Desearía agradecer al lic. J. Osores, por haber permitido esta investigación, de igual forma a la Ing. Paula Olivera por hacer posible las mediciones por FRX.

\section{Referencias}

[1] UNSCEAR, (1993). United Nations Scientific Committee on the effect of atomic radiation. Sources and effects of ionizing radiation. Report to the General Assembly with scientific Annexes. New York: United Nations, 45.

[2] Evans, R. D. (1969). Engineers guide to the elementary behavior of radon daughters. Health Physics Pergamon Press. Vol. 17, pp. 229-252.

[3] Nazaroff, W.W. y A. V. Nero (1988). Radon and its decay products in indoor air. John Wiley \& Sons, USA, pp 1-30.

[4] Haque, A.K.M.M. y E. Kirk, (1992). Environmental Radon and Cancer Risk. Radiation Protection Dosimetry Vol 4S N $1 / 4$ nuclear technology Publishing, USA pp. 639642.

[5] San Miguel, E.G., J. P. Pérez, J. P. Bolívar, R. García. Determinación de ${ }^{238} \mathrm{U}$ en sedimentos por espectrometría Gamma. Departamento de Física aplicada, EPS La Rábida 21819-Palos, Huelva, España.

[6] Quindós, L. S., J. Soto y Fernández (2000). Measurements the exhalation rate of $222 \mathrm{Rn}$ from solid samples. Huelva España.

[7] Environmental Implementation Guide for Radiological Survey Procedures February 1997 U.S. Department of Energy Assistant Secretary for Environment, Safety and Health Washington, D.C. 20585. 